

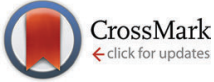

Cite this: Soft Matter, 2017, 13, 1940

Received 25th October 2016 Accepted 18th January 2017

DOI: $10.1039 / c 6 s m 02413 k$

rsc.li/soft-matter-journal

\section{Concepts for soft interfaces}

\author{
Reinhard Sigel \\ Concepts and opportunities of interfaces with soft properties are discussed. Such interfaces show a \\ strong response to external fields. How can the interface tension which governs interfacial behavior \\ become compatible with soft and fluctuating degrees of freedom at the interface?
}

Many technical applications of soft materials rely on the large response of these materials to rather weak forces. The bestknown examples are the easy deformation of rubbers and non glassy polymeric materials, and the change of liquid crystalline orientation by moderate electric fields. These large responses also occur by the action of thermal energy $k_{\mathrm{B}} T$, and thus thermal fluctuations of significant magnitude are common in soft matter systems. The properties of large response and significant thermal fluctuations are referred to as soft degrees of freedom. ${ }^{1}$ On the other hand, many properties of Soft Matter cannot be considered as soft degrees of freedom, because they show little difference to the behavior of hard matter. An example is the density, where tiny values of the compressibility indicate minor changes upon a pressure variation. The assumption of incompressible soft matter is usually a very good approximation.

One motivation for the formation of the SOMATAI (Soft Matter at Aqueous Interfaces) Marie Currie Initial Training Network came from posing the question, of whether the characteristic strong response of soft matter to external stimuli and the resulting fluctuations can be realized at interfaces. The properties of an interface with such a soft degree of freedom could be switched by weak external fields. In systems with large internal interfaces like emulsions, the soft interfacial degrees of freedom could significantly affect the overall material performance.

The concept of a soft interface is related to the notion of 'interfaces and interphases'. This homophone pair appears to originate from adhesion science, ${ }^{2-4}$ and has been applied in different context. ${ }^{5,6}$ In this notion, the transition zone of finite width between two phases is addressed as a separate entity having its own character. This 'interphase' is not a true phase in the thermodynamic sense, but it is not a purely two dimensional separation sheet between the two neighbouring bulk

Max-Planck-Institut für Eisenforschung GmbH, 40237 Düsseldorf, Germany.

E-mail: res314159@aol.de phases either. The layer between the bulk phases can be affected by external fields. Here we look for concepts to increase its response.

Interface tension plays the role of the major antagonist against interface fluctuations. It aims to keep the interface area as small as possible. Interface bound fluctuations of large thermal amplitude must not significantly affect the interface area. Consequently, only interfacial degrees of freedom which are weakly coupled to the interface area can become soft, except for low interface tension systems. ${ }^{7-9}$ The inverse interface tension corresponds to the low compressibility in bulk systems. In bulk, only fluctuations which do not involve compression acquire large thermal amplitudes.

It is interesting here to take a look at hard interfaces. A recent workshop organized by Peter Fischer (ETH Zürich) on dynamics of complex fluid-fluid interfaces at Monte Verità, Ascona (Switzerland) contained many contributions on interfacial rheology. ${ }^{10}$ The majority of investigated systems had rather complicated interface structure and rheology, with interfacial aggregates of surfactants which where stiff and chunky. The two dimensional excluded volume repulsion of these bulky islands created non-homogeneous interface coverage. Although not stated explicitly in this workshop, it appears that stiff or meta-stable in-plane structures in the interfaces are employed to improve the stability of emulsions. For this task with many applications in food science and other areas, the specific creation of hard interfaces appears to be an advantage. It is an interesting concept, to stabilize thermodynamic metastable emulsions by non-ergodic interface structures. A combination of stiff islands or metastable structures with other soft degrees of freedom at the interface appears challenging. For soft interfaces, it might be better to suppress solid-like interfacial aggregates. For this purpose, the usage of surfactants with less regular chemical structure, e.g. with unsaturated hydro carbon chains, where crystallization is hindered, appears to be of advantage. 
An inspection of soft matter concepts for bulk material can advance the search for soft interfacial degrees of freedom, specifically, on discussion of how these concepts can be transferred to interfaces. A notion which addresses directly the name of the Soft Matter field was introduced by Peter Pusey. ${ }^{11} \mathrm{He}$ considers colloidal particles as soft matter because an interaction strength equivalent to $k_{\mathrm{B}} T$ for rather large particles leads to a low shear modulus of a mesoscopic model fluid formed by the colloidal particles. The argument is well adapted to colloid physics, where the colloidal system usually is tuned to mimic hard spheres or sticky hard spheres for a good comparison with computer simulations of a similar system. The focus of colloid physics is thus reduced to a corner of the parameter space, where charge-charge interactions have a short screening length and contrast in refractive index is matched, in order to minimize van der Waals attraction. The Pusey corner of the colloidal domain, where interface physics is reduced to effective hard sphere or effective sticky hard sphere conditions, is opposite to the aim discussed here to develop soft interfaces and to implement interesting interphases.

It is worth critically reviewing Pusey's argument. An interaction strength with a magnitude not higher than $k_{\mathrm{B}} T$ is required for colloidal stability. However, it does not follow from first principles; the system is tuned to such low interactions. Stronger opposite interactions like the van der Waals attraction and the repulsion due to electrostatic or steric stabilization almost compensate each other. The physics of the colloidal mesoscopic fluid thus occurs neither on a different energy scale, nor - in general - on a different length scale than the underlying interface physics of colloidal stabilization. A separation of the two appears arbitrary and might hamper a deep understanding of colloidal systems. For the creation of soft interfaces, the most valuable idea of the Pusey notion is the compensation of opposite interactions stronger than $k_{\mathrm{B}} T$. An example is colloidal particles trapped at an interface. Capillary interaction might first reorient the particles, but then in general is attractive. ${ }^{12,13}$ The strong attraction needs a compensation, e.g. by electrostatic repulsion of charged particles.

A second concept for soft matter relies on phase transitions and spontaneous symmetry breaking. Close to a second order or weakly first order phase transition, the amplitude and the relaxation time of thermal fluctuations become large, and the system shows large response to external fields. A well-known example from bulk systems is the nematic to isotropic phase transition of a liquid crystal. A promising concept for the realization of soft interfaces starts from interface phase transitions where the parameters are tuned close to a second order phase transition. The interface rheology for anisotropic particles incorporated in an interface could be switched if the range of orientation correlation is changed by an interface phase transition, e.g. by a change of temperature or interaction strength. Beside a complete immersion of anisotropic particles in the interface, anisotropic molecules could be anchored to an interface via surfactants and spacers. The table of contents figure for this contribution visualizes such a system. A transition similar to a smectic A to smectic C lyotropic system where the tilt angles of the anisotropic molecules relative to the interface normal are changed breaks the symmetry and induces anisotropic behaviour within the interface. For the spherical colloidal particles at an interface mentioned above, the phase transitions have been well characterized for magnetically controlled interactions. ${ }^{14-16}$ In a similar way, the interaction of charged particles which stabilize a Pickering emulsion ${ }^{17,18}$ could be tuned to a second order phase transition by a variation of the screening length. The interface fluctuations and response functions could then strongly affect the overall behavior of the emulsion. Another interface phase transition is the pre-wetting transition for a two phase system (see e.g. ref. 1), where for conditions close to critical pre-wetting a strong effect of external fields is to be expected. For an orientational pre-wetting layer at a solid-liquid crystalline interface, fluctuations of the interphase have been detected experimentally. ${ }^{19,20}$

A further soft matter class are polymers. An overview of the extensive scientific activities on polymers at interfaces is far beyond the possibilities of this contribution. Polymer brushes have attracted much attention. ${ }^{21,22}$ Responsive brushes have been reviewed by Minko. ${ }^{23}$ In many cases, related work investigates structural changes of the brushes only. The concept of interfacial softness might contribute to shift the attention towards the change of interfacial properties and relaxation times for such responsive systems. The interface analogue of semidilute polymer solutions which are known for strong concentration fluctuations in bulk are polymers adsorbed flat to an interface. The confinement of polymers to two dimensions might be realized with a polymer which carries on each monomer a hydrophilic group and a hydrophobic group, so a polysoap. ${ }^{24-26}$ The two dimensional adsorption of such polymers without defects appears challenging from a preparatory point of view. In case sufficient mobility of the molecules could be retained, such a system would allow the fluctuations of a semidilute polymer solution in the two dimensions of the interface, with related strong responses to external fields.

In conclusion, the concept of interfacial softness and ergodicity is a fruitful guiding idea for the design and the physical understanding of interesting new materials. Strong interface response functions to external fields can be realized by a transfer of concepts known from bulk soft matter to interfaces. For internal interfaces in emulsions and colloidal systems, it might be challenging to realize soft interfacial degrees of freedom while keeping emulsion or colloidal stability. An experimental characterization of such systems requires investigations on structure, properties, and dynamics.

The author thanks all PIs and PhD students of the EU FP7 Marie Curie Initial Training Network SOMATAI (Grant 316866) for stimulating discussions and exchange. Furthermore, the author thanks Andreas Erbe for support. Helgard Sigel is gratefully acknowledged for her hospitality.

\section{References}

1 R. Sigel, in Interfaces of Binary Mixtures, ed. P. R. Lang and Y. Liu, Springer International Publishing, Cham, 2016, pp. 221-278. 
2 L. T. Drzal, in The interphase in epoxy composites, ed. K. Dušek, Springer Berlin Heidelberg, Berlin, Heidelberg, 1986, pp. 1-32.

$3 \mathrm{~J}$. D. Miller and H. Ishida, in Adhesive-Adherend Interface and Interphase, ed. L.-H. Lee, Springer US, Boston, MA, 1991, pp. 291-324.

4 L. T. Drzal, in Adhesive-Adherend Interface and Interphase, ed. D. Miracle and S. Donaldson, ASM International, 2001, pp. 169-179.

5 K. E. Geckeler, F. Rupp and J. Geis-Gerstorfer, Adv. Mater., 1997, 9, 513-518.

6 G. J. Dvorak, Micromechanics of Composite Materials, Springer Dordrecht Heidelberg, Dordrecht, Heidelberg, 2013.

7 E. Scholten, R. Tuinier, R. H. Tromp and H. N. W. Lekkerkerker, Langmuir, 2002, 18, 2234-2238.

8 D. G. A. L. Aarts, M. Schmidt and H. N. W. Lekkerkerker, Science, 2004, 304, 847-850.

9 D. G. A. L. Aarts and H. N. W. Lekkerkerker, J. Fluid Mech., 2008, 606, 275-294.

10 P. Fischer, L. Isa, A. Studart, L. Sagis and J. Vermant, (Organizers), Dynamics of complex fluid-fluid interfaces, workshop at the Monte Verità, Ascona, Switzerland, 13.18.7.2014. The booklet of abstracts is available at http:// www.fpe.ethz.ch/dcffi-2014.html.

11 P. N. Pusey, in Colloidal Suspensions, ed. J. P. Hansen, D. Levesque and J. J. Zinn-Justin, Elsevier, Amsterdam, 1991, pp. 765-942.
12 J. C. Loudet, A. M. Alsayed, J. Zhang and A. G. Yodh, Phys. Rev. Lett., 2005, 94, 018301.

13 L. Botto, E. P. Lewandowski, M. Cavallaro and K. J. Stebe, Soft Matter, 2012, 8, 9957-9971.

14 U. Gasser, C. Eisenmann, G. Maret and P. Keim, ChemPhysChem, 2010, 11, 963-970.

15 P. Dillmann, G. Maret and P. Keim, J. Phys.: Condens. Matter, 2012, 24, 464119.

16 S. Deutschländer, P. Dillmann, G. Maret and P. Keim, Proc. Natl. Acad. Sci. U. S. A., 2015, 112, 6925-6930.

17 M. Oettel and S. Dietrich, Langmuir, 2008, 24, 1425-1441.

18 P. A. Kralchevsky, K. D. Danov and P. V. Petkov, Philos. Trans. R. Soc., A, 2016, 374, 20150130.

19 R. Sigel and G. Strobl, Prog. Colloid Polym. Sci., 1997, 104, 187-190.

20 R. Sigel and G. Strobl, J. Chem. Phys., 2000, 112, 1029-1039.

21 R. C. Advincula, W. J. Brittain, K. C. Caster and J. Ruehe, Polymer Brushes: Synthesis, Characterization, Applications, Springer Dordrecht Heidelberg, Dordrecht, Heidelberg, 2004.

22 O. Azzaroni, J. Polym. Sci., Part A: Polym. Chem., 2012, 50, 3225-3258.

23 S. Minko, J. Macromol. Sci., Part C: Polym. Rev., 2006, 46, 397-420. 24 A. Laschewsky, Adv. Polym. Sci., 1995, 124, 1-86.

25 S. Garnier, A. Laschewsky and J. Storsberg, Tenside, Surfactants, Deterg., 2006, 43, 88-102.

26 P. Raffa, D. A. Z. Wever, F. Picchioni and A. A. Broekhuis, Chem. Rev., 2015, 115, 8504-8563. 